\title{
Cystatin $\mathbf{C}$ and Frailty Status in Older Men
}

\author{
Allyson Hart, MD, MS ${ }^{1}$, Misti L. Paudel, MPH${ }^{2}$, Brent C. Taylor, PhD, MPH ${ }^{1,2,3}$, Areef Ishani, \\ MD, MS ${ }^{1,4}$, Eric S. Orwoll, MD ${ }^{5}$, Peggy M. Cawthon, PhD $^{6}$, Kristine E. Ensrud, MD, MPH ${ }^{1,2,3}$, \\ and for the Osteoporotic Fractures in Men (MrOS) Study Group \\ ${ }^{1}$ Department of Medicine, University of Minnesota, Minneapolis, MN \\ ${ }^{2}$ Division of Epidemiology \& Community Health, University of Minnesota, Minneapolis, MN \\ ${ }^{3}$ Center for Chronic Disease Outcomes Research, Minneapolis VA Health Care System, \\ Minneapolis, MN \\ ${ }^{4}$ Department of Nephrology, Minneapolis VA Health Care System, Minneapolis, MN \\ ${ }^{5}$ Bone and Mineral Unit, Oregon Health \&Science University, Portland, OR \\ ${ }^{6}$ California Pacific Medical Center Research Institute, San Francisco, CA
}

\section{Abstract}

OBJECTIVES-Declining kidney function and frailty are common with aging, but the association between these conditions remains uncertain. We hypothesized that higher cystatin $\mathrm{C}$ would be associated with greater frailty status in men age 65 years and older.

DESIGN—Cross-sectional cohort study

SETTING AND PARTICIPANTS-Men $\succeq 65$ years from six U.S. sites

MEASUREMENTS-Serum cystatin C and creatinine were measured along with frailty status in a random sample of 1602 community-dwelling men age $\ 65$ years enrolled in the MrOS study. Frailty status was analyzed as an ordinal outcome of robust, intermediate stage, and frail using a multinomial logistic regression model and the base model was adjusted for age, race, and clinical site.

RESULTS-The mean age of the cohort was 73.8 years; $9.8 \%$ were frail and $47.2 \%$ were intermediate stage. Higher cystatin $\mathrm{C}$ was associated with a 7 -fold higher odds of frail vs. robust status (OR quartile 4 vs. 1; 7.12, 95\% CI 3.76-13.46) and a 2.4-fold higher odds of intermediate vs. robust status (OR quartile 4 vs. $1 ; 2.38,95 \%$ CI 1.70-3.32). The association was attenuated but

\footnotetext{
Corresponding author: Allyson Hart, MD, MS, Division of Renal Diseases and Hypertension, 717 Delaware Street, S.E., Suite 353, Mail Delivery Code 1932, Minneapolis, MN 55414, Phone: (612) 624-9444, Fax: (612) 626-3840, hart1044@umn.edu.

These data were presented in a poster at the National Kidney Foundation annual meeting in Washington DC, May 2012.

Conflict of Interest:

Drs. Orwoll and Ensrud have received grant support from the NIH (and supporting agencies) grant as listed under Funding Sources on the title page. Dr. Ensrud also serves as a consultant for Merck Sharpe \& Dohme on a Data Monitoring Committee.

Author Contributions:

Allyson Hart, MD, MS - analysis and interpretation of data, preparation of manuscript

Misti L. Paudel, MPH - analysis and interpretation of data, critical review of manuscript

Brent C. Taylor, PhD, MPH - interpretation of data, critical review of manuscript

Areef Ishani, MD, MS - interpretation of data, critical review of manuscript

Eric S. Orwoll, MD - study concept and design, acquisition of subjects and data, interpretation of data, critical review of manuscript Peggy M. Cawthon, $\mathrm{PhD}$ - interpretation of data, critical review of manuscript

Kristine E. Ensrud, MD, MPH - study concept and design, acquisition of subjects and data, interpretation of data, critical review of manuscript
} 
persisted after adjusting for multiple possible confounders. In contrast, neither higher serum creatinine (OR quartile 4 vs. 1; 1.36, 95\% CI 0.78-2.40) nor lower creatinine-based eGFR (OR quartile 4 vs. $1 ; 1.01,95 \%$ CI $0.54-1.87$ ) was associated in a graded manner with higher odds of frailty.

CONCLUSION-Higher cystatin C, but not creatinine-based measures, was associated with increased odds of frailty status in this cohort of older men.

\section{Keywords}

Cystatin C; creatinine; frailty; elderly; kidney function

\section{INTRODUCTION}

Decreased kidney function is common in the elderly, with more than $30 \%$ of those aged 70 years and older having an estimated glomerular filtration rate (eGFR) of less than $60 \mathrm{ml} /$ $\mathrm{min} / 1.73 \mathrm{~m}^{2} .{ }^{1}$ Older adults with an eGFR of less than $60 \mathrm{ml} / \mathrm{min} / 1.73 \mathrm{~m}^{2}$ have an increased risk of subsequent cardiovascular disease and death, but the association between mild to moderate renal dysfunction and other adverse health outcomes in the aged population is uncertain.

Clinicians caring for older adults have long recognized a syndrome of frailty manifested by decreased physiologic reserve and increased vulnerability to physical decline and death. In 2001, Fried and colleagues using data collected in the cardiovascular health study (CHS) proposed a standard definition of in which three or more of the following criteria are present: shrinking (e.g., unintentional weight loss), self-reported exhaustion or poor energy, weakness manifested by low grip strength, slow walking speed and low physical activity. Subjects with none of the criteria are considered to be robust, while those with one or two criteria are considered to be in an intermediate, or pre-frail, stage. ${ }^{2}$ In their analysis, frailty defined by this index was predictive of increased risk of falling, hospitalization, disability and mortality. While individuals with disabilities and comorbidities were more likely to be classified as frail, $27 \%$ of subjects had neither disability nor comorbidity, and frailty status was predictive of poor outcomes independent of these other factors, suggesting a physiologic basis for the phenotype described. $2 ; 3$ Although several other instruments have been developed to operationalize the construct of frailty, the CHS index has been most extensively studied and the predictive value of this index has been validated in multiple cohorts. ${ }^{4-8}$

While frailty is common among older adults with advanced or end-stage kidney disease, how mild to moderate age-related deficits in kidney function relate to frailty is less extensively studied. The association between kidney function and frailty in older adults was previously evaluated in the cardiovascular health study (CHS) cohort using serum creatinine as the measure of kidney function. ${ }^{9}$ In this cross-sectional analysis, higher creatinine $(>1.3$ $\mathrm{mg} / \mathrm{dL}$ in women and $>1.5 \mathrm{mg} / \mathrm{dL}$ in men) was associated with higher odds of frailty. However, serum creatinine may not be an accurate measure of measure of kidney function in older individuals with decreased muscle mass and these cutoff values of creatinine likely represented markedly reduced kidney function. ${ }^{10 ; 11}$ In addition, frailty status was expressed as a dichotomous outcome (frail versus not frail) in this analysis, not as a continuum ranging from robust to intermediate stage to frail.

Cystatin C, a lower molecular weight protein whose concentration is mainly determined by glomerular filtration, is a measure of kidney function that is less dependent on muscle mass. ${ }^{12 ; 13}$ Thus, cystatin $\mathrm{C}$ may be superior to standard creatinine-based measures in detecting mild to moderate reductions in kidney function in older adults unselected for 
kidney disease. ${ }^{14 ; 15}$ To test the hypothesis that higher cystatin C levels are associated with greater prevalent frailty status, we measured cystatin $\mathrm{C}$ and assessed frailty status in a cohort of 1602 community dwelling men aged $\searrow 65$ years enrolled in the Osteoporotic Fractures in Men (MrOS) study. In addition, we examined whether other measures of reduced kidney function, including higher serum creatinine or lower creatinine-based estimated glomerular filtration rate, were associated with frailty status.

\section{METHODS}

\section{Participants}

The Osteoporotic Fractures in Men (MrOS) Study is a prospective multi-center observational study of a cohort of 5994 community-dwelling men aged 65 or older, able to walk without assistance and without history of bilateral hip replacements recruited between 2000 and 2002 from six clinical sites: Minneapolis, Birmingham, Palo Alto, Pittsburgh, Portland, and San Diego. Detailed descriptions of the study design and recruitment for MrOS have been previously published. ${ }^{16 ; 17}$ A random sample of 1604 men were selected for measurement of cystatin $\mathrm{C}$, and of these, 1602 men had adequate had sufficient sera for performance of cystatin $\mathrm{C}$ assay and comprised the analytical cohort. The institutional review board (IRB) at each center approved the protocol and written informed consent was obtained from all participants.

\section{Biochemical Data}

Fasting morning blood was collected at the baseline examination and processed for serum which was stored at $-70^{\circ} \mathrm{C}$ until thawed. Serum cystatin $\mathrm{C}$ assays were performed at the University of Minnesota Medical Center in 2010. Serum cystatin C concentrations were determined using a BN100 nephelometer (Dade Behring Inc., Deerfield, IL) using a particleenhanced immunonepholometric assay ${ }^{18}$ (assay range $0.23-8.00 \mathrm{mg} / \mathrm{L}$ with inter-assay coefficient of variation (CV) of $4.0 \%$ at a level of $0.71 \mathrm{mg} / \mathrm{L}$ and $3.1 \%$ at a level of $1.75 \mathrm{mg} /$ $\mathrm{L}$ [mean inter-assay CV 3.7\%]) and then converted to standardized values traceable to a certified reference material. ${ }^{19}$ Cystatin C-based eGFR (eGFR cysC $_{\text {C }}$, was computed using a CKD-EPI equation re-expressed for standardized cystatin C. 19;20

Serum creatinine was measured using the Roche 911 analyzer (Roche diagnostic Corporation, Indianapolis, IN) at the Portland VA Medical Center using the Jaffe rateblanked method calibrated with materials assayed by isotope-dilution mass spectrometry (IDMS). Inter-assay $\mathrm{CV}$ was $4.0 \%$. Creatinine-based eGFR (eGFR $\mathrm{Cr}_{\mathrm{r}}$ ) using was calculated using the chronic kidney disease epidemiology collaboration (CKD-EPI) equation ${ }^{21}$, which includes variables for standardized creatinine, age, gender and race. Serum albumin was measured using a Roche COBAS Integra 800 automated analyzer (Roche Diagnostic Corporation, Indianapolis, IN) with inter-assay CV of $1.98 \%$. Measures of $25(\mathrm{OH}) \mathrm{D}_{2}$ and $25(\mathrm{OH}) \mathrm{D}_{3}$ were performed at the Mayo Clinic using liquid chromatography-mass spectrometry and summed for total 25(OH)D. Total intact parathyroid hormone (PTH) was measured using an immunoradiometric assay (Scantibodies Laboratory, Inc. Santee, CA) at the Columbia University Laboratory. Markers of chronic inflammatory processes were assessed by serum level of the pro-inflammatory cytokine IL-6 using a high-sensitivity ELISA from R\&D systems (Minneapolis, $\mathrm{MN}$ ) and by serum levels of high sensitivity $\mathrm{C}$ reactive protein (CRP) measured using the BNII nephelometer (Dade Behring Inc., Deerfield, IL) utilizing a particle-enhanced immunonephelometric assay.

\section{Clinic Measurements}

At the baseline examination, height was measured with Harpenden stadiometers and weight was measured on balance beam or digital scales. Body mass index (BMI) was calculated as 
$\mathrm{kg} / \mathrm{m}^{2}$. Appendicular skeletal mass was measured using dual energy $\mathrm{x}$-ray absorptiometry (DXA) on Hologic QDR4500 machines (Hologic, Inc. Bedford, MA) using standardized protocols. Walking speed was measured on a six meter walking course with participants asked to walk at their usual pace, and expressed as meters/second. The fastest pace of two trials was recorded. Grip strength $(\mathrm{kg})$ was measured using a Jamar hand dynamometer (Lafayette Instrument Co. Lafayette IN).

\section{Questionnaire Data}

Demographic information, social support, smoking status, and alcohol use were ascertained through self-administered questionnaires. Men were asked whether they had received a physician diagnosis of the following medical: hypo- or hyperthyroidism, transient ischemic attack or stroke, diabetes mellitus, hypertension, coronary heart disease or myocardial infarction, angina, congestive heart failure, chronic obstructive pulmonary disease, intermittent claudication, liver disease, Parkinsonism, or cancer (except non-melanoma skin cancer). Perceived health status was self-reported as "excellent," "good," "fair," "poor," or "very poor" in response to the question "Compared to other people your own age, how would you rate your overall health?" Physical activity was assessed using the Physical Activity Scale for the Elderly (PASE) score. ${ }^{22}$

\section{Frailty Status Assessment}

Frailty status was defined using a modified CHS frailty index ${ }^{6}$ comprised of the following five components: first, shrinking, defined by the lowest quintile of appendicular lean mass at the baseline examination, adjusted for height and body fat. Second, weakness, identified by grip strength in the lowest quintile stratified by body mass index quartiles. Third, exhaustion, identified by an answer of "little or none" to the question "How much of the time during the past 4 weeks did you have a lot of energy" from the Medical Outcomes Survey Short Form, a 12-item questionnaire at the baseline examination. Fourth, slowness, identified by a walk speed at the baseline examination in the lowest quintile stratified by for standing height quartile. Fifth, low physical activity level, identified by a PASE score in the lowest quintile. Men with none of the five components were classified as robust, those with 1 or 2 components were classified as intermediate stage, and those with $\geq 3$ components classified as frail. Measurements used to define the components of shrinking, poor energy, and low physical activity were similar but not identical to those used in the original phenotype proposed (see Supplementary Table 1). ${ }^{6 ; 7}$

\section{Statistical Analysis}

Differences in baseline characteristics by quartile of cystatin $\mathrm{C}$ were compared using chisquare (or categorical data) and ANOVA (for normally-distributed continuous data) tests.

For the analysis, Cystatin $\mathrm{C}$ and creatinine-based predictors were expressed as quartiles, to ensure adequate numbers of subjects and preserve power. In secondary analyses, we expressed eGFR predictors in categories using clinically relevant cutpoints corresponding to the presence or absence of CKD of $<60 \mathrm{ml} / \mathrm{min} / 1.73 \mathrm{~m}^{2}$ and $\succ 60 \mathrm{ml} / \mathrm{min} / 1.73 \mathrm{~m}^{2}$. Further categorization of eGFR $<60 \mathrm{ml} / \mathrm{min} / 1.73 \mathrm{~m}^{2}$ was not performed due to inadequate numbers of subjects in these categories.

Given the ordinal nature of the frailty status outcome variable (robust, intermediate stage or frail), multinomial logistic regression was used to simultaneously calculate the odds of being frail versus robust and intermediate status versus robust. Cystatin $\mathrm{C}$ was expressed as quartiles, with the lowest quartile serving as the referent group. Crude models were performed, then adjusted for age, race and clinical site (base model). In forming the multivariate model, the pre-specified covariates that may be related to cystatin $\mathrm{C}$ level or 
frailty status (body mass index, self-reported health status, number of comorbid conditions, alcohol consumption, smoking status, social support, independent activities of daily living (IADL) impairments, education, use of an angiotensin blockage agent, vitamin D levels, intact PTH levels, and serum albumin) were added individually to the base model to assess for significance as independent predictors of frailty status at a significance level of alpha $=$ 0.05 , as well as potential confounding defined as changing the frailty odds parameter estimates by at least $10 \%$. Sensitivity analyses were also performed adjusting for individual comorbid conditions (history of stroke, myocardial infarction, angina, congestive heart failure, fracture, non-skin cancer, diabetes, hypertension, COPD, Parkinson's, hypo- or hyperthyroidism) to assess for the presence of residual confounding potentially masked by adjustment for number of conditions. Covariates that were either independent predictors or potential confounders were included in the final multivariable model. A test for linear trend was performed by expressing cystatin $\mathrm{C}$ quartile as an ordinal variable. We also performed analyses examining the association of the presence of CKD by eGFR (eGFR $<60 \mathrm{ml} / \mathrm{min} /$ $1.73 \mathrm{~m}^{2}$ ), defined using both cystatin $\mathrm{C}$ and creatinine-based equations, and frailty status.

To examine the biological mechanisms that might underlie the association observed between cystatin $\mathrm{C}$ and frailty status, levels of calcitropic hormones $(25(\mathrm{OH}) \mathrm{D}, \mathrm{iPTH})$ and markers of chronic inflammation (TNF-a, IL-6, TNF-asR1, TNF-asR2, IL-6SR, and CRP) were added individually to the base model. To further evaluate the effect of specific inflammatory markers, (CRP, IL-6) on the association, a test of interaction between cystatin $\mathrm{C}$ and each of these markers for prediction of frailty status was performed. To evaluate whether the association between cystatin $\mathrm{C}$ and frailty status was driven by one of the five components of frailty, the association between cystatin $\mathrm{C}$ and each individual component of frailty (e.g., shrinking, weakness, exhaustion, slowness or low physical activity) was evaluated using a multiple logistic regression model. All analyses were conducted using SAS version 9.2 (SAS inc., Cary, NC).

\section{RESULTS}

Baseline characteristics of the analytical cohort $(\mathrm{n}=1602)$ and by quartile of cystatin $\mathrm{C}$ are shown in Table 1. The mean age of the cohort was 73.8 years and $91.5 \%$ of the cohort selfidentified as Caucasian. 157 (9.8\%) subjects in the cohort were classified as frail while 756 (47.2\%) were classified as intermediate status. 1252 (78.4\%) had a cystatin C-based CKDEPI eGFR $\geq 60 \mathrm{ml} / \mathrm{min} / 1.73 \mathrm{~m}^{2} ; 329(20.4 \%)$ had an eGFR $30-59 \mathrm{ml} / \mathrm{min} / 1.73 \mathrm{~m}^{2}$, and only $21(1.3 \%)$ had an eGFR $<30 \mathrm{ml} / \mathrm{min} / 1.73 \mathrm{~m}^{2}$. Using creatinine-based CKD-EPI eGFR, 1257 $(83.5 \%)$ had an eGFR $\searrow 60 \mathrm{ml} / \mathrm{min} / 1.73 \mathrm{~m}^{2}, 236(15.7 \%)$ had an eGFR of $30-59 \mathrm{ml} / \mathrm{min} /$ $1.73 \mathrm{~m}^{2}$, and $12(0.8 \%)$ had an eGFR $<30 \mathrm{ml} / \mathrm{min} / 1.73 \mathrm{~m}^{2}$. The correlation of cystatin C (and $\mathrm{eGFR}_{\mathrm{cys}}$ ) with eGFR $\mathrm{Cr}_{\mathrm{r}}$ was moderate in magnitude (Spearmann Correlation Coefficient $=0.66$ ). Higher cystatin $\mathrm{C}$ was associated with older age, greater number of comorbidities and greater impairments in independent activities of daily living.

After adjustment for age, site and race, higher cystatin $\mathrm{C}$ was associated with greater odds of being classified as intermediate stage (versus robust) and frail (versus robust). Compared to men in the lowest quartile of cystatin C (referent), men in quartile four had a seven-fold greater odds of frailty (OR 7.1, 95\% CI 3.76-13.46), and a greater than two-fold greater odds of intermediate status (OR 2.4, 95\% CI 1.70-3.32) (Table 2). The associations appeared graded in nature ( $\mathrm{p}$ for linear trend across cystatin $\mathrm{C}$ quartiles $<0.001$ ). The associations were attenuated but persisted after adjusting for multiple covariates including functional impairment and number of comorbid conditions; compared to men in the first quartile of cystatin $\mathrm{C}$ (referent), men in quartile four had a four-fold greater odds of frailty (OR 4.17, 95\% CI 2.02-8.59), and a 2-fold greater odds of intermediate status (OR 2.1, 95\% 1.44-2.98). In addition to cystatin $\mathrm{C}$, age $(\mathrm{p}<0.001)$, self-reported health status $(\mathrm{p}<0.001)$ 
and number of IADL impairments ( $\mathrm{p}<0.001)$ were most predictive of frailty status. Findings about the association between higher cystatin $\mathrm{C}$ and greater frailty status were not altered in sensitivity analyses adjusting for the presence of specific comorbid medical conditions. The association between cystatin $\mathrm{C}$ and frailty status also persisted when the analysis was restricted to the 1257 subjects without chronic kidney disease as defined by a creatininebased estimated GFR using the CKD-EPI equation of $₫ 60 \mathrm{ml} / \mathrm{min} / 1.73 \mathrm{~m}^{2}$ (see Supplementary Table 2).

After adjustment for age, race and site, higher cystatin $\mathrm{C}$ was associated with greater odds of each individual frailty component, including higher odds of shrinking, weakness, exhaustion, slowness and low physical activity (Table 3). After consideration of multiple confounders, associations between higher cystatin $\mathrm{C}$ and a greater likelihood of shrinking, slowness and low physical activity remained significant.

The association between eGFR $\mathrm{cysC}_{\mathrm{c}}$ and frailty status was not explained by alterations in calcitropic hormones (iPTH and $25(\mathrm{OH}) \mathrm{D}$ ), albumin or inflammatory markers (Table 4). Further adjustment of the base model for each of the potential biological mediators had little impact on the association, though adjustment for IL-6 reduced the magnitude of the association by more than $30 \%$. There was no evidence for interaction between cystatin C level and IL-6 ( $p>0.8$ ). Results from analyses adjusting for TNF- $\alpha$, IL-10, TNF-asR1, TNF-asR2, or IL-6SR (results not shown) were similar to those from analyses adjusted for CRP. Conversely, neither higher serum creatinine quartile ( $\mathrm{p}$-value for trend across quartiles of creatinine $>0.4$ ) nor lower estimated GFR quartile calculated using the serum creatininebased CKD-EPI formula (p-trend across quartiles of eGFR $\mathrm{Cr}_{\mathrm{r}}>0.6$ ) was associated with higher odds of frailty. Substitution of $\mathrm{GFFR}_{\mathrm{Cr}}$ calculated by the MDRD equation for of eGFR $_{\mathrm{Cr}}$ calculated using the CKD-EPI equation did not change the results. When the lowest quartile of creatinine-based eGFR $\left(\mathrm{eGFR}_{\mathrm{Cr}}<64.7 \mathrm{ml} / \mathrm{min} / 1.73 \mathrm{~m}^{2}\right.$ ) was compared to the highest three quartiles, the lack of an association persisted (results not shown). However, the presence of chronic kidney disease as identified by a creatinine-based eGFR $<60 \mathrm{ml} / \mathrm{min} /$ $1.73 \mathrm{~m}^{2}$ (CKD-EPI formula) was associated with higher odds of both intermediate and frail status, though the associations between chronic kidney disease and frailty status appeared stronger in magnitude when a cystatin-C based equation was used to calculate eGFR (Table $5)$.

\section{DISCUSSION}

In this cohort of older men, higher cystatin $\mathrm{C}$ was strongly associated with greater prevalent frailty status, and this association was independent of several potential confounders and biologic mediators. Higher cystatin $\mathrm{C}$ was also associated with higher odds of each of the individual components of frailty with no single component appearing to drive the association. In contrast, higher serum creatinine and lower eGFR $\mathrm{Cr}_{\mathrm{r}}$ were not associated in a graded manner with a higher odds of greater frailty status. The presence of CKD defined using either a cystatin-C based or creatinine-based equation was associated with a higher likelihood of greater frailty status, confirming results reported by Wilhelm-Leen et al that examined CKD as defined by eGFR $\mathrm{Cr}<60 \mathrm{ml} / \mathrm{min} / 1.73 \mathrm{~m}^{2}$ as a predictor of frailty in a younger NHANES population. ${ }^{23}$ However, the association between markers of kidney function and frailty was much stronger in magnitude in this cohort for CKD defined using a cystatin-C based equation as compared with that for CKD defined using a creatinine-based equation.

These findings are consistent with previous studies that have found cystatin $\mathrm{C}$ to be predictive of other measures of unsuccessful aging, although the definitions and metrics used to define this outcome have varied. A cross-sectional study of the Health, Aging, and 
Body Composition (HABC) cohort reported that higher cystatin C was independently associated with poorer performance on objective tests of physical performance. ${ }^{24} \mathrm{In}$ contrast, lower serum creatinine-based estimated glomerular filtration rate (eGFR) was not associated with poorer performance. Longitudinal analysis of the HABC cohort showed cystatin $\mathrm{C}$ to be predictive of self-reported physical functional limitations. ${ }^{25} \mathrm{In}$ a longitudinal study of the CHS cohort, higher cystatin C was also associated with decreased aging success, using various definitions including a composite of reported comorbid conditions, cognitive impairment and self-reported physical impairments. ${ }^{26}$ Recently, Roshanravan et al reported that higher cystatin $\mathrm{C}$ concentration was associated with a higher prevalence of frailty, although the study examined younger population selected for the presence of CKD and did not evaluate frailty as a continuum from robust to intermediate stage to frail. ${ }^{27}$ To our knowledge, this is the first study to evaluate the association of kidney function as assessed by both cystatin $\mathrm{C}$ and creatinine-based measures with the outcome of frailty status in an elderly population unselected for kidney disease.

The mechanism linking higher cystatin $C$ to frailty remains unclear. Increased levels of inflammatory markers have been associated with decreased kidney function ${ }^{28}$ as well as with frailty. ${ }^{3 ; 29}$ Inflammation may also increase the level of cystatin C. ${ }^{30}$ However, we found no evidence for interaction between inflammatory markers and cystatin $\mathrm{C}$ for the prediction of frailty status. In addition, after controlling for these markers, the association between cystatin $\mathrm{C}$ and frailty persisted, suggesting that the association cannot be fully explained by higher levels of inflammation among those men with elevated cystatin C levels. Higher cystatin $\mathrm{C}$ was also associated with each of the individual components of frailty, suggesting that a single component such as shrinking or physical activity was not driving the association noted between cystatin $\mathrm{C}$ and frailty as a syndrome. Other potential biologic mediators such as intact PTH, vitamin D and albumin also had little or no effect on the association between cystatin $\mathrm{C}$ and frailty, nor did adjustment for medical comorbidities, health status and IADL impairments.

In contrast to cystatin $\mathrm{C}$, we found no evidence of a graded association of serum creatinine or eGFR $\mathrm{Cr}_{\mathrm{r}}$ (expressed in quartiles) with frailty status. A prior study using data collected in CHS reported a significant association between serum creatinine and frailty, but used a cutoff of $1.3 \mathrm{mg} / \mathrm{dL}$ in women and $1.5 \mathrm{mg} / \mathrm{dL}$ in men to define elevated creatinine. ${ }^{9}$ This cutoff likely represents a marked reduction in kidney function in an elderly population with decreased muscle mass; our findings suggest that cystatin $\mathrm{C}$ is strongly predictive of frailty status at even "normal" levels of kidney function. The relationship between creatinine (and eGFR $_{\mathrm{Cr}}$ ) quartile and frailty status appeared J-shaped in this study, such that men in both the highest and lowest quartiles of creatinine and $\mathrm{eGFR}_{\mathrm{Cr}}$ had a higher odds of frailty, albeit not at the level of signficance. This J-shaped pattern between creatinine or $\mathrm{eGFR}_{\mathrm{Cr}}$ and frailty may reflect the dependence of serum creatinine level and $\mathrm{eGFR}_{\mathrm{Cr}}$ on muscle mass.

We found that CKD as defined by either creatinine or cystatin C was associated with an increased odds of intermediate or frail status, though the association between CKD defined by cystatin $\mathrm{C}$ and frailty status was almost twice as strong as that between CKD defined by serum creatinine and frailty status. The contrasting results when using cystatin $\mathrm{C}$ versus creatinine suggest that cystatin $\mathrm{C}$ may be a more sensitive marker of mild to moderate, but prognostically significant, decreases in kidney function in an elderly population, a hypothesis supported by the additional finding that cystatin $\mathrm{C}$ was predictive of frailty status even when the analysis was restricted to subjects whose eGFR calculated using serum creatinine was $\measuredangle 60 \mathrm{ml} / \mathrm{min} / 1.73 \mathrm{~m}^{2}$. Furthermore, the prevalence of CKD in this population was higher using a cystatin C-based formula to calculate eGFR compared to that when using a creatinine-based formula to calculate eGFR ( $21.6 \%$ versus $16.5 \%$, respectively). 
This study has several limitations. Most importantly, this cross-sectional evaluation can only suggest an association between higher cystatin $\mathrm{C}$ and greater frailty status. Future prospective studies examining the association between baseline cystatin $\mathrm{C}$ among non-frail older adults and risk of incident frailty are needed. We relied on surrogate markers of kidney function rather than direct measurements. While cystatin $\mathrm{C}$ may be a better marker of kidney function in older adults due to its lower dependence on muscle mass, this remains an area in need of further study. MrOS is a cohort of ambulatory, community-dwelling, predominantly Caucasian men, and findings may not be generalizable to other population groups; however, these results are consistent with other studies in different populations such as younger populations and populations selected on the basis of CKD. Very few men at the MrOS baseline examination had advanced CKD or proteinuria, limiting our ability to assess the association between cystatin $\mathrm{C}$ and frailty in those with more significant kidney function impairment. Components in the modified CHS frailty index used in this study were similar but not identical to those in the CHS frailty index. However, previous studies have reported that the associations between this modified frailty index ${ }^{6 ; 7}$ and risk of adverse health outcomes are similar in magnitude to those between the CHS frailty index and risk of adverse outcomes. Finally, as with any observational study, the possibility for residual confounding persists.

In conclusion, higher cystatin $\mathrm{C}$ was associated with increased odds of greater frailty status in older men. Cystatin C (and CKD defined using a cystatin C-based equation) is a better predictor of frailty status than traditional creatinine-based measures of renal function. These findings suggest that higher cystatin $\mathrm{C}$ may be a promising biomarker for unsuccessful aging as defined by frailty. Further studies are needed to establish whether cystatin $\mathrm{C}$ predicts the development of incident frailty, and to further elucidate whether this association is present because cystatin $\mathrm{C}$ is a more sensitive and specific marker of decreased kidney function in the elderly, or whether it is reflective of factors other than reduced renal function.

\section{Supplementary Material}

Refer to Web version on PubMed Central for supplementary material.

\section{Acknowledgments}

Support and Financial Disclosure

Support: The Osteoporotic Fractures in Men (MrOS) Study is supported by National Institutes of Health funding. The following institutes provide support: the National Institute of Arthritis and Musculoskeletal and Skin Diseases (NIAMS), the National Institute on Aging (NIA), the National Center for Research Resources (NCRR), and NIH Roadmap for Medical Research under the following grant numbers: U01 AR45580, U01 AR45614, U01 AR45632, U01 AR45647, U01 AR45654, U01 AR45583, U01 AG18197, U01-AG027810, and UL1 RR024140.

Sponsor's Role: The funding agencies had no direct role in the conduct of the study; the collection, management, analyses and interpretation of the data; or preparation or approval of the manuscript.

\section{REFERENCES}

1. Coresh J, Selvin E, Stevens LA, et al. Prevalence of chronic kidney disease in the United States. JAMA. 2007; 298:2038-2047. [PubMed: 17986697]

2. Fried LP, Tangen CM, Walston J, et al. Frailty in older adults: evidence for a phenotype. J Gerontol A Biol Sci Med Sci. 2001; 56:M146-M156. [PubMed: 11253156]

3. Walston J, Mcburnie MA, Newman A, et al. Frailty and activation of the inflammation and coagulation systems with and without clinical comorbidities: Results from the Cardiovascular Health Study. Arch Intern Med. 2002; 162:2333-2341. [PubMed: 12418947] 
4. Bandeen-Roche K, Xue QL, Ferrucci L, et al. Phenotype of frailty: characterization in the women's health and aging studies. J Gerontol A Biol Sci Med Sci. 2006; 61:262-266. [PubMed: 16567375]

5. Boyd CM, Xue QL, Simpson CF, et al. Frailty, hospitalization, and progression of disability in a cohort of disabled older women. Am J Med. 2005; 118:1225-1231. [PubMed: 16271906]

6. Cawthon PM, Marshall LM, Michael Y, et al. Frailty in older men: Prevalence, progression, and relationship with mortality. J Am Geriatr Soc. 2007; 55:1216-1223. [PubMed: 17661960]

7. Ensrud KE, Ewing SK, Cawthon PM, et al. A comparison of frailty indexes for the prediction of falls, disability, fractures, and mortality in older men. J Am Geriatr Soc. 2009; 57:492-498. [PubMed: 19245414]

8. Woods NF, LaCroix AZ, Gray SL, et al. Frailty: Emergence and consequences in women aged 65 and older in the Women's Health Initiative Observational Study. J Am Geriatr Soc. 2005; 53:13211330. [PubMed: 16078957]

9. Shlipak MG, Stehman-Breen C, Fried LF, et al. The presence of frailty in elderly persons with chronic renal insufficiency. Am J Kidney Dis. 2004; 43:861-867. [PubMed: 15112177]

10. Finney H, Bates CJ, Price CP. Plasma cystatin C determinations in a healthy elderly population. Arch Gerontol Geriatr. 1999; 29:75-94. [PubMed: 15374079]

11. Wasen E, Isoaho R, Mattila K, et al. Estimation of glomerular filtration rate in the elderly: A comparison of creatinine-based formulae with serum cystatin C. J Intern Med. 2004; 256:70-78. [PubMed: 15189368]

12. Baxmann AC, Ahmed MS, Marques NC, et al. Influence of muscle mass and physical activity on serum and urinary creatinine and serum cystatin C. Clin J Am Soc Nephrol. 2008; 3:348-354. [PubMed: 18235143]

13. Westhuyzen J, Cystatin C. A promising marker and predictor of impaired renal function. Ann Clin Lab Sci. 2006; 36:387-394. [PubMed: 17127725]

14. Fliser D, Ritz E. Serum cystatin C concentration as a marker of renal dysfunction in the elderly. Am J Kidney Dis. 2001; 37:79-83. [PubMed: 11136171]

15. Schaeffner ES, Ebert N, Delanaye P, et al. Two novel equations to estimate kidney function in persons aged 70 years or older. Ann Intern Med. 2012; 157:471-481. [PubMed: 23027318]

16. Blank JB, Cawthon PM, Carrion-Petersen ML, et al. Overview of recruitment for the osteoporotic fractures in men study (MrOS). Contemp Clin Trials. 2005; 26:557-568. [PubMed: 16085466]

17. Orwoll E, Blank JB, Barrett-Connor E, et al. Design and baseline characteristics of the osteoporotic fractures in men (MrOS) study--a large observational study of the determinants of fracture in older men. Contemp Clin Trials. 2005; 26:569-585. [PubMed: 16084776]

18. Erlandsen EJ, Randers E, Kristensen JH. Evaluation of the Dade Behring N Latex Cystatin C assay on the Dade Behring Nephelometer II System. Scand J Clin Lab Invest. 1999; 59:1-8. [PubMed: 10206092]

19. Inker LA, Eckfeldt J, Levey AS, et al. Expressing the CKD-EPI (Chronic Kidney Disease Epidemiology Collaboration) cystatin C equations for estimating GFR with standardized serum cystatin C values. Am J Kidney Dis. 2011; 58:682-684. [PubMed: 21855190]

20. Stevens LA, Coresh J, Schmid CH, et al. Estimating GFR using serum cystatin C alone and in combination with serum creatinine: a pooled analysis of 3,418 individuals with CKD. Am J Kidney Dis. 2008; 51:395-406. [PubMed: 18295055]

21. Levey AS, Stevens LA, Schmid CH, et al. A new equation to estimate glomerular filtration rate. Ann Intern Med. 2009; 150:604-612. [PubMed: 19414839]

22. Washburn RA, Smith KW, Jette AM, et al. The Physical Activity Scale for the Elderly (PASE): Development and evaluation. J Clin Epidemiol. 1993; 46:153-162. [PubMed: 8437031]

23. Wilhelm-Leen ER, Hall YN, Tamura K, et al. Frailty and chronic kidney disease: the Third National Health and Nutrition Evaluation Survey. Am J Med. 2009; 122:664-671. [PubMed: 19559169]

24. Odden MC, Chertow GM, Fried LF, et al. Cystatin C and measures of physical function in elderly adults: The Health, Aging, and Body Composition (HABC) Study. Am J Epidemiol. 2006; 164:1180-1189. [PubMed: 17035344] 
25. Fried LF, Lee JS, Shlipak M, et al. Chronic kidney disease and functional limitation in older people: Health, aging and body composition study. J Am Geriatr Soc. 2006; 54:750-756. [PubMed: 16696739]

26. Sarnak MJ, Katz R, Fried LF, et al. Cystatin C and aging success. Arch Intern Med. 2008; 168:147-153. [PubMed: 18227360]

27. Roshanravan B, Khatri M, Robinson-Cohen C, et al. A prospective study of frailty in nephrologyreferred patients with CKD. Am J Kidney Dis. 2012; 60:912-921. [PubMed: 22770927]

28. Shlipak MG, Fried LF, Crump C, et al. Elevations of inflammatory and procoagulant biomarkers in elderly persons with renal insufficiency. Circulation. 2003; 107:87-92. [PubMed: 12515748]

29. Hamerman D. Toward an understanding of frailty. Ann Intern Med. 1999; 130:945-950. [PubMed: 10375351]

30. Stevens LA, Schmid CH, Greene T, et al. Factors other than glomerular filtration rate affect serum cystatin C levels. Kidney Int. 2009; 75:652-660. [PubMed: 19119287] 


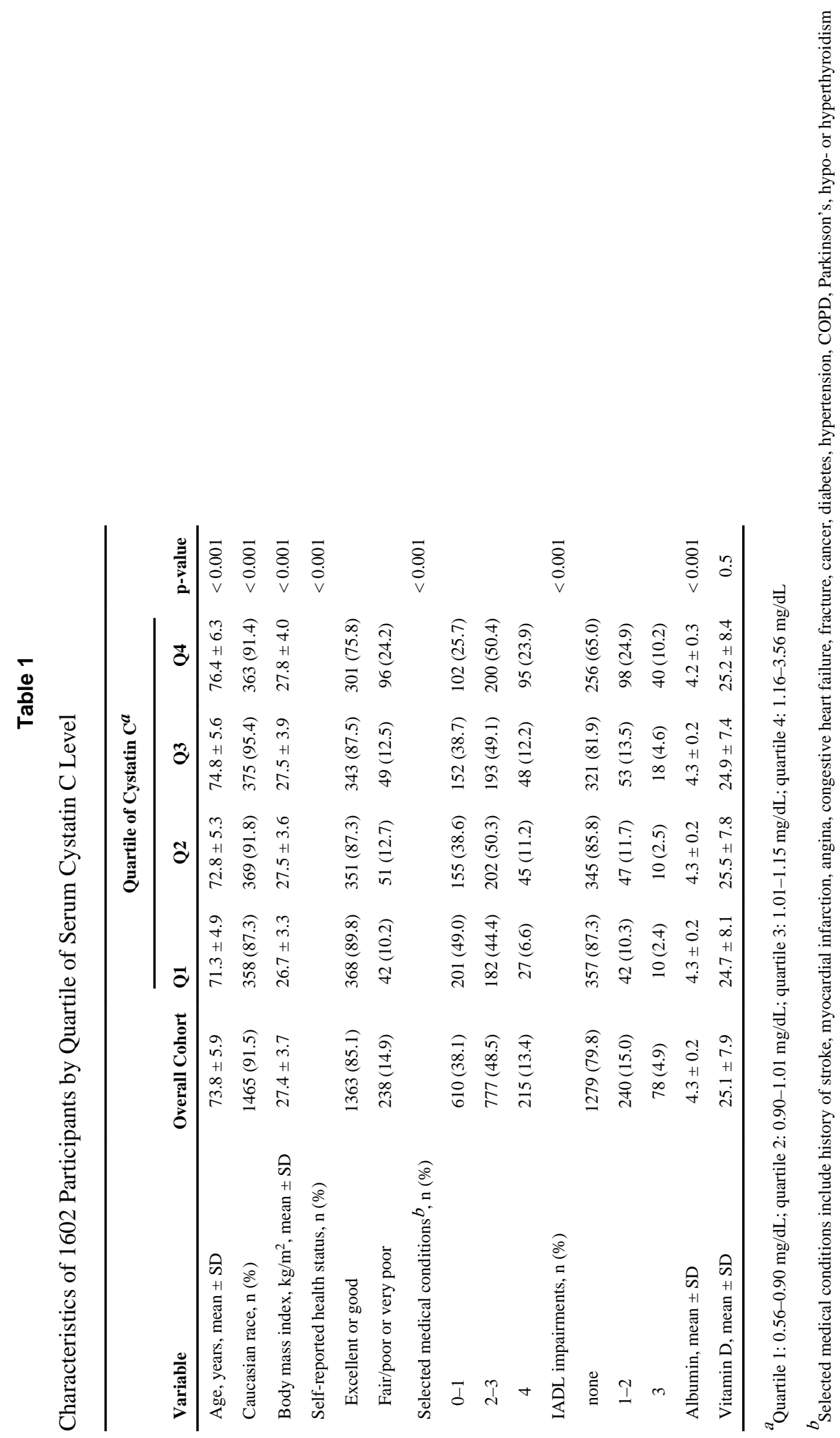


Table 2

Association between Measures of Kidney Function and Odds of Frailty Status

\begin{tabular}{|c|c|c|c|c|}
\hline \multirow[b]{3}{*}{ Measure of Kidney Function $c$} & \multicolumn{4}{|c|}{ Multinomial Odds Ratio (95\% Confidence Interval) } \\
\hline & \multicolumn{2}{|c|}{ Base Model $^{a}$} & \multicolumn{2}{|c|}{ 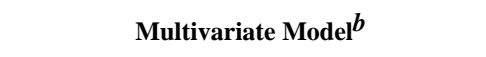 } \\
\hline & Intermediate vs. Robust & Frail vs. Robust & Intermediate vs. Robust & Frail vs. Robust \\
\hline \multicolumn{5}{|l|}{ Cystatin C } \\
\hline Quartile 1 & 1.00 (referent) & 1.00 (referent) & 1.00 (referent) & 1.00 (referent) \\
\hline Quartile 2 & $1.26(0.94-1.69)$ & $1.98(1.01-3.88)$ & $1.23(0.90-1.69)$ & $1.86(0.88-3.89)$ \\
\hline Quartile 3 & $1.15(0.85-1.56)$ & $1.66(0.84-3.28)$ & $1.13(0.81-1.56)$ & $1.31(0.61-2.81)$ \\
\hline Quartile 4 & $2.38(1.70-3.32)$ & $7.12(3.76-13.46)$ & $2.07(1.44-2.98)$ & $4.17(2.02-8.59)$ \\
\hline p-trend & $<0.001$ & $<0.001$ & $<0.001$ & $<0.001$ \\
\hline \multicolumn{5}{|l|}{ Creatinine } \\
\hline Quartile 1 & 1.00 (referent) & 1.00 (referent) & 1.00 (referent) & 1.00 (referent) \\
\hline Quartile 2 & $0.81(0.58-1.14)$ & $0.61(0.33-1.11)$ & $0.89(0.64-1.25)$ & $0.70(0.36-1.37)$ \\
\hline Quartile 3 & $0.73(0.54-0.99)$ & $0.46(0.26-0.80)$ & $0.78(0.57-1.07)$ & $0.57(0.31-1.06)$ \\
\hline Quartile 4 & $1.11(0.77-1.59)$ & $1.36(0.78-2.40)$ & $1.11(0.76-1.61)$ & $1.21(0.65-2.27)$ \\
\hline p-trend & 0.96 & 0.43 & 0.87 & 0.49 \\
\hline \multicolumn{5}{|l|}{ eGFR (CKD-EPI Cr) } \\
\hline Quartile 4 & 1.00 (referent) & 1.00 (referent) & 1.00 (referent) & 1.00 (referent) \\
\hline Quartile 3 & $1.10(0.79-1.53)$ & $0.61(0.33-1.16)$ & $1.27(0.90-1.78)$ & $0.99(0.49-2.00)$ \\
\hline Quartile 2 & $0.97(0.70-1.33)$ & $0.59(0.32-1.11)$ & $1.04(0.75-1.45)$ & $0.95(0.48-1.91)$ \\
\hline Quartile 1 & $1.12(0.79-1.59)$ & $1.01(0.54-1.87)$ & $1.12(0.77-1.61)$ & $1.11(0.56-2.23)$ \\
\hline p-trend & 0.87 & 0.49 & 0.92 & 0.67 \\
\hline
\end{tabular}




\section{Table 4}

Effect of Biological Mediators on the Association between Cystatin C eGFR $<60$ ml/min/1.73m² and Frailty

\begin{tabular}{lccc}
\hline & & \multicolumn{2}{c}{ Odds Ratio (95\% CI) ${ }^{\boldsymbol{a}}$} \\
\cline { 3 - 4 } & $\mathbf{n}$ & Intermediate vs. Robust & Frail vs. Robust \\
\hline Base model $b$ & 974 & $2.12(1.43-3.14)$ & $5.32(3.00-9.43)$ \\
Base model + IL-6 & 974 & $1.82(1.21-2.73)$ & $3.75(2.04-6.88)$ \\
Base model + CRP & 952 & $2.07(1.39-3.09)$ & $5.00(2.80-8.93)$ \\
Base model + vit D & 969 & $2.17(1.47-3.22)$ & $5.67(3.17-10.13)$ \\
Base model + iPTH & 969 & $2.06(1.39-3.06)$ & $5.02(2.80-8.99)$ \\
Base model + albumin & 949 & $2.16(1.44-3.25)$ & $4.39(2.33-8.27)$ \\
\hline
\end{tabular}

Reference: eGFR $\Varangle 60 \mathrm{ml} / \mathrm{min} / 1.73 \mathrm{~m}^{2}$

${ }^{b}$ Adjusted for age, race and clinical site 
Table 5

Association between CKD (eGFR <60) and Odds of Frailty Status Measured by Cystatin C and Creatinine

\begin{tabular}{|c|c|c|c|c|}
\hline \multirow[b]{3}{*}{ Measure of Renal Function } & \multicolumn{4}{|c|}{ Multinomial Odds Ratio (95\% Confidence Interval) } \\
\hline & \multicolumn{2}{|c|}{ Base Model } & \multicolumn{2}{|c|}{ Multivariate Model } \\
\hline & Intermediate vs. Robust & Frail vs. Robust & Intermediate vs. Robust & Frail vs. Robust \\
\hline \multicolumn{5}{|l|}{ Cystatin C eGFR (CKD-EPI) } \\
\hline$\checkmark 60 \mathrm{ml} / \mathrm{min} / 1.73 \mathrm{~m}^{2}$ & 1.00 (referent) & 1.00 (referent) & 1.00 (referent) & 1.00 (referent) \\
\hline$<60 \mathrm{ml} / \mathrm{min} / 1.73 \mathrm{~m}^{2}$ & $2.21(1.64-2.99)$ & $4.63(3.03-7.08)$ & $1.88(1.36-2.60)$ & $2.76(1.68-4.56)$ \\
\hline \multicolumn{5}{|l|}{ Creatinine eGFR (CKD-EPI) } \\
\hline$\checkmark 60 \mathrm{ml} / \mathrm{min} / 1.73 \mathrm{~m}^{2}$ & 1.00 (referent) & 1.00 (referent) & 1.00 (referent) & 1.00 (referent) \\
\hline$<60 \mathrm{ml} / \mathrm{min} / 1.73 \mathrm{~m}^{2}$ & $1.70(1.21-2.40)$ & $2.56(1.59-4.12)$ & $1.59(1.11-2.27)$ & $1.91(1.11-3.27)$ \\
\hline
\end{tabular}

